\title{
Effect of the Japanese herbal medicine, Boiogito, on the osteoarthritis of the knee with joint effusion
}

Tokifumi Majima ${ }^{*}$, Masahiro Inoue², Yasuhiko Kasahara², Tomohiro Onodera², Daisuke Takahashi ${ }^{2}$ and Akio Minami

\begin{abstract}
Background: Boiogito (Japanese herbal medicine, Tsumura Co. Tokyo, Japan) contains sinomenin which inhibits inflammatory reactions. Since sinomenine is a principle component of the Boiogito, there is a possibility of it being effective on osteoarthritis (OA) of the knee with joint effusion. However, there is no report concerning the effectiveness of Boiogito on knee OA. The objective of the present study is to investigate the therapeutic effect of Boiogito on OA of the knee associated with joint effusion in a comparative study among randomly assigned groups.

Methods: Study was performed using 50 patients who were diagnosed with primary osteoarthritis of the knee with joint effusion. The patients were randomly assigned to two groups: one group (25 patients) using both loxoprofen (2-\{4-[(2-oxocyclopentyl) methyl]\} propanoic acid) and Boiogito and the other group (25 patients) using loxoprofen, and were evaluated during a 12 week observation period. The assessment parameters including knee scores in the Knee Society Rating System including Knee score and Functional scores, amount of joint effusion by joint puncture in clinically detected cases, the 36-items short form of the Medical Outcome Study Questionnaire (SF-36) as a measurement of health related quality of life were used.

Results: The knee scores based on the Knee Society Rating System were improved in both groups. The staircase climbing up and down ability in the Knee society rating system functional score was significantly improved in the group using Boiogito and loxoprofen compared to the loxoprofen group. In the evaluation using SF-36, significant improvements were found in the scores in both groups in physical functioning after 12 weeks. The amount of joint fluid was significantly decreased at 4, 8 and 12 weeks compared to pre-administration baseline in the group using Boiogito and loxoprofen. A side effect of Boiogito, dry mouth, was found in one case. The symptom was mild and improved immediately after discontinuation of administration.
\end{abstract}

Conclusion: The results indicated that Boiogito have a possibility for a treatment modality for joint effusion with osteoarthritis of the knee.

Keywords: Osteoarthritis of the knee, Boiogito, Japanese herbal medicine, joint effusion

\section{Introduction}

Osteoarthritis $(\mathrm{OA})$ of the knee is a degenerative disease of the knee joints which significantly damages the functions of knee joints. The objective of its treatment is to decrease pain while attempting to maintain or increase the range of knee motion and to minimize disabilities in

\footnotetext{
* Correspondence: tkmajima@med.hokudai.ac.jp

'Department of Joint Replacement and Tissue Engineering, Hokkaido

University Graduate School of Medicine, Sapporo, Japan

Full list of author information is available at the end of the article
}

daily living. The majority of osteoarthritic patients visit the clinic with the complaint of pain. As clinical symptoms, joint effusion is frequently associated with pain and limitation in the range of knee motion.

As conservative therapies for OA of the knee, education and physical therapy, oral administration of nonsteroidal anti-inflammatory drugs (NSAIDs), intraarticular injection of hyaluronic acid, and use of lateral wedge insole or unloading braces are applied based on the degrees of symptoms [1]. Further, diacerein, which 
is one kind of herbal medicine, has been used clinically as a symptom modifying drug for reducing joint pain in Europe and the United States of America [2]. It has been reported that diacerein has the chondromodulating effect on the hip joint in observating radiographs among OA patients of the hip joint in a total of 507 cases [3]. Further, diacerein was reported to be effective on OA of the knee [4]. In Asia, the alkaloid, sinomenine is extracted from the Chinese medical plant Sinomeniuim acutum, which has been utilized by Chinese doctors for over 2000 years to treat various rheumatic diseases [5]. Previous pharmacological studies have demonstrated that sinomenine inhibits inflammatory reactions $[5,6]$.

Boiogito (Japanese herbal medicine, Tsumura Co., Tokyo, Japan) contains a dry extract $3.75 \mathrm{~g}$ of the mixed drug substance consisting of Sinomenium Stem $5.0 \mathrm{~g}$, Astragalus Root 5.0 g, Atractylodes Lancea Rhizome 3.0 g, Jujube $3.0 \mathrm{~g}$, Glycyrrhiza $1.5 \mathrm{~g}$, and Ginger $1.0 \mathrm{~g}$ in a daily dose of $7.5 \mathrm{~g}$. That is constituted of numerous components including sinomenine as a principal component (Figure 1). Since sinomenine is a principle component, there is a possibility of it being effective on OA of the knee with joint effusion. The objective of the present study is to investigate the therapeutic effect of Boiogito on OA of the knee associated with joint effusion in a comparative study among randomly assigned groups.

\section{Materials and methods}

The subjects used were patients with primary OA of the knee associated with clinically detected joint effusion. Those were outpatients having pain in the knee joints while walking and showing a Kellgren-Lawrence [7] grade III or less in the radiographs. The patients having serious complications (liver diseases, kidney diseases, hematological diseases, etc.), those incapable of taking oral administration, those currently taking other herbal

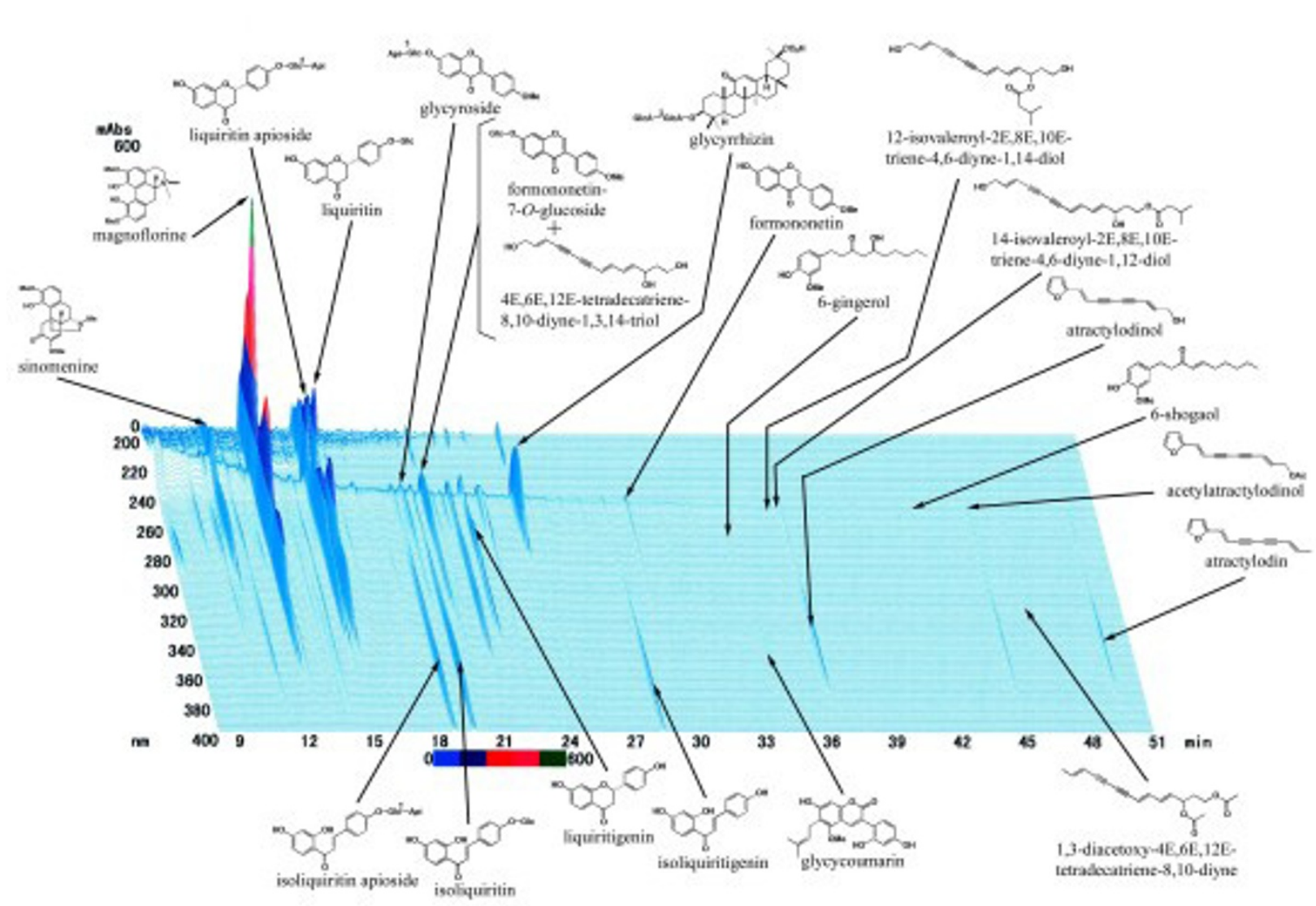

Figure 1 Three Dimensional pattern of High Performance Liquid Chromatography in Boiogito. HPLC apparatus consisted of a Shimadzu LC 10A (analysis system software: CLASS-M10A ver. 1.64, Tokyo, Japan) equipped with a multiple wavelength detector (UV 200-400 nm) (Shimadzu SPD-M10AVP, diode array detector), an auto injector (Shimadzu CTO-10AC). HPLC conditions were described as follows: column, ODS (TSK-GEL 80TS, $250 \times 4.6$ mm i.d., TOSOH, Tokyo, Japan); eluent, (A) 0.05 M AcONH4 (pH 3.6) (B) $100 \%$ CH3CN. A linear gradient of 90\% A and $10 \%$ B changing over 60 min to $0 \%$ A and 100\% B was used. And 100\% B was continued for 20 min.; temperature, 40 degrees celusius; flow rate, $1.0 \mathrm{~mL} / \mathrm{min}$. A granule was extracted with methanol $(20 \mathrm{~mL})$ under ultrasonication for $30 \mathrm{~min}$., and was centrifuged at $3000 \mathrm{rpm}$ for $5 \mathrm{~min}$. The supernatant was filtrated with a membrane filter $(0.45 \mu \mathrm{m})$ and then submitted for HPLC analysis $(30 \mu \mathrm{L})$. 
medications and those having a hyaluronic acid injection to the joint were excluded from the study. After approval of the Institutional Review Board at the Hokkaido University Hospital, a random, blinded intergroup comparison study was conducted.

The subjects were randomly divided into two groups: one group treated by concomitant use of Boiogito and loxoprofen (thereinafter referred to as the concomitant use group) and loxoprofen administration group (hereinafter referred to as the loxoprofen group). Prior to the study, the subjects signed a written consent of their own free will. The treatment drug, Boiogito, was administered at a daily dose of $7.5 \mathrm{~g}$ divided into three portions which were given before meals or between meals. Further, loxoprofen at a single dose of $60 \mathrm{mg}$ was orally administered after meals three times daily. The number of subjects enrolled in the present study was 50 subjects divided into 25 subjects in the concomitant use group and 25 subjects in the loxoprofen group with respect to the treatment drugs. Among them, three cases including two cases (loxoprofen group) who did not come back after the initial examination and one case (concomitant use group) who did not come back after $4^{\text {th }}$ week were excluded from the study. As a result, the remaining 47 subjects (24 cases in the concomitant use group and 23 cases in the loxoprofen group) were used in the analysis of the treatment results.

The period of administration of the treatment drug was 12 weeks. The assessment parameters including knee scores in the Knee Society Rating System including Knee score and Functional scores [8], amount of joint effusion by joint puncture in clinically detected cases, the 36-items short form of the Medical Outcome Study Questionnaire (SF-36) as a measurement of health related quality of life were used. Further, the side effects were investigated.
For statistical analyses, according to the types of data, the following methods were used: $\chi^{2}$ tests for ratio of male and female, Wilcoxon's T- test for patients background, change in SF-36 and Knee Society Rating System knee score and functional scores, one-way analysis of variance for change in volume of joint fluid, and then, a Bonferroni analysis was applied to determine the significance of difference for multiple comparison. Significant level was set at $\mathrm{p}<0.05$.

\section{Results}

Regarding the patients' background, with respect to gender in both groups, the number of females was greater than the number of males. The mean age was 68.3 years in the concomitant use group and 71.5 years in the loxoprofen group. The detailed preoperative comparison between the 2 groups is shown in Table 1 . There were no significant differences in preoperative factors between the two groups.

The knee score in the Knee Society Rating System was $75.0+/-15.1$ before administration in the concomitant use group which improved to $85.8+/-11.1$ at the time of final observation and $72.8+/-16.0$ before administration in the loxoprofen group which improved to 81.7 $+/$ - 15.1, demonstrating significant improvements in both groups $(\mathrm{p}<0.05)$. The staircase climbing up and down ability in the Knee Society Rating System of functional score significantly improved from $32.2+/-6.3$ before administration to $40.2+/-4.4$ at the time of final observation in the concomitant use group $(p<0.05)$. In the loxoprofen group, the functional score of stairs in Knee Society Rating System changed from 28.4 +/- 6.4 before administration to $36.6+/-6.2$ at the final follow up. This change has no significant difference.

In the evaluation of SF-36 which is an assessment of the quality of life of the patients, significant

Table 1 Patient demographics

\begin{tabular}{|c|c|c|c|}
\hline & $\begin{array}{l}\text { Concomitant use group (Loxoprofen and } \\
\text { Boiogito) }\end{array}$ & $\begin{array}{l}\text { Loxoprofen group } \\
\text { (Loxoprofen) }\end{array}$ & $\begin{array}{l}\mathrm{p}- \\
\text { value }\end{array}$ \\
\hline Number of patients & 24 & 23 & - \\
\hline Sex Female & 19 & 18 & - \\
\hline Male & 5 & 5 & - \\
\hline Average age & $68.3+/-10.0$ & $71.5+/-6.0$ & N.S \\
\hline Height (cm) & $154.0+/-6.9$ & $152.5+/-6.2$ & N.S \\
\hline Weight (kg) & $61.7+/-6.2$ & $62.3+/-5.7$ & N.S \\
\hline Average period of symptoms & $14.5+/-30$ & $25.0+/-23.1$ & N.S \\
\hline Knee score in Knee Society Rating System & $75.0+/-15.1$ & $72.8+/-16.0$ & N.S \\
\hline $\begin{array}{l}\text { Functional score in Knee Society Rating } \\
\text { System }\end{array}$ & $32.2+/-6.3$ & $28.4+/-6.4$ & N.S \\
\hline \multirow[t]{2}{*}{ Number of Kellgren-Lawrence Grade } & II: 2 & II: 2 & - \\
\hline & ||l:22 & ||l:21 & \\
\hline
\end{tabular}

Data is shown as mean and standard deviation. 
improvements were detected in both groups in the physical functions after 12 weeks $(\mathrm{p}<0.05)$ (Boiogito and loxoprofen administration group in Figure 2, Loxoprofen administration group in Figure 3).

In terms of quantity of joint effusion, the quantity of joint effusion was an average of $15.1+/-7.1 \mathrm{ml}$ in the concomitant use group and an average of $12.1+/-5.7$ $\mathrm{ml}$ in the loxoprofen group when punctured before the treatment. There was no significant difference in quantity of joint effusion between two groups before administration. The amounts of joint effusion at the times of 4 weeks, 8 weeks and 12 weeks after treatment were significantly lower compared to those prior to the treatment in the concomitant use group $(\mathrm{p}<0.05)$. On the other hand, there were no significant differences in the amount of joint effusion at the time of 4,8 , and 12 weeks after treatment compared to those before treatment in the loxoprofen group (Figure 4).

As a side effect which seemed to be caused by administration of Boiogito, one case of "dry mouth" was reported. The symptom was mild and was improved immediately after discontinuation of administration.

\section{Discussion}

OA of the knee is a degenerative disease which onsets mostly among the elderly. The number of osteoarthritic patients is simply increasing in society due to the increasing number of the elderly population. The clinical symptoms of OA of the knee include pain as well as limitation in the range of knee motion and joint effusion. As the OA of the knee advances, the symptoms cause a significant effect on activities of daily living.

When the degeneration of knee joints is not severe, conservatinve treatment including drug therapies and physical therapies are selected. For pain management, NSAIDs are widely used. It has been reported that

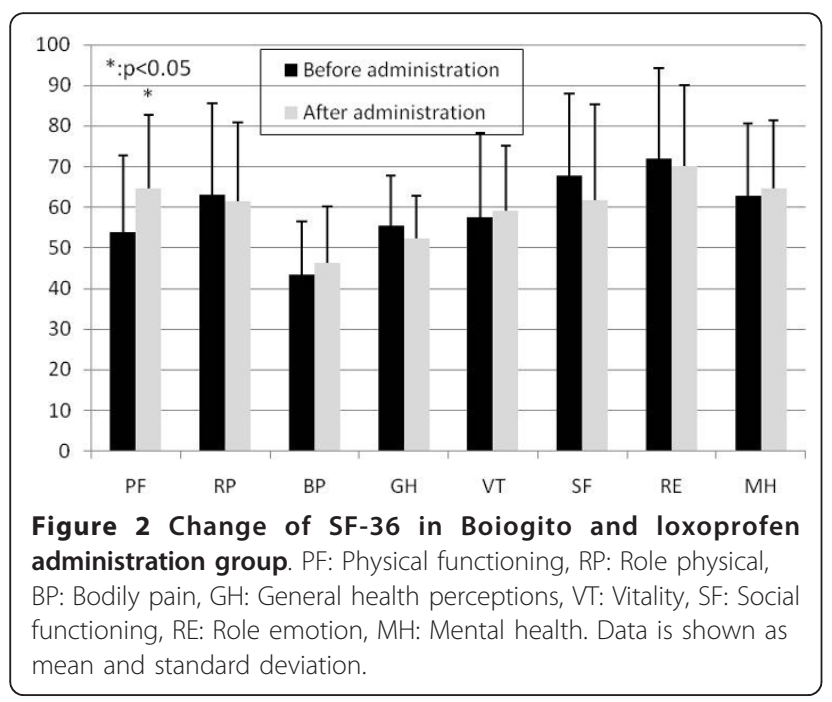

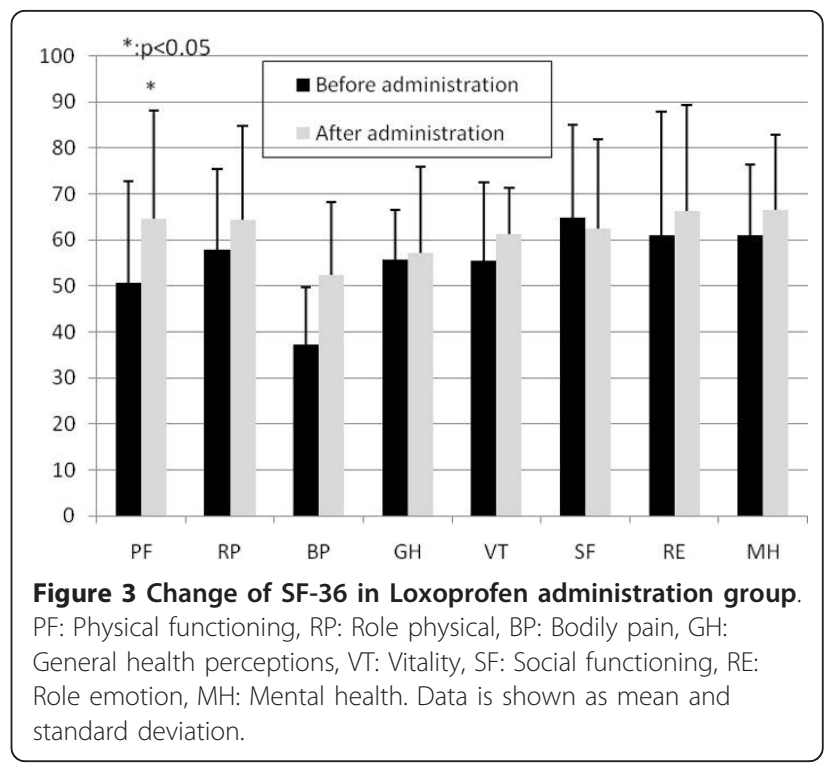

NSAIDs have a major role in the management of OA in a systematic review $[9,10]$. On the other hand, problems such as an inhibitory action of long-term application of NSAIDs on proteoglycan synthesis of cartilage was pointed out $[11,12]$. In some cases, joint effusion exists in spite of various treatments. The joint effusion in the knee contains many aggrecan fragments and MMP-1. It has been reported that joint effusion has adverse effects on the articular cartilage $[13,14]$. According to the results of the present study, joint effusion in OA of the knee significantly decreased by using Boiogito along with NSAIDs. In addition, staircase climbing up and down ability in the knee society score and Physical functioning of SF-36 were found to be improved significantly. These phenomena were assumed to be related to the fact that systematic conditions were improved as a result of improvement in joint effusion by Boiogito.

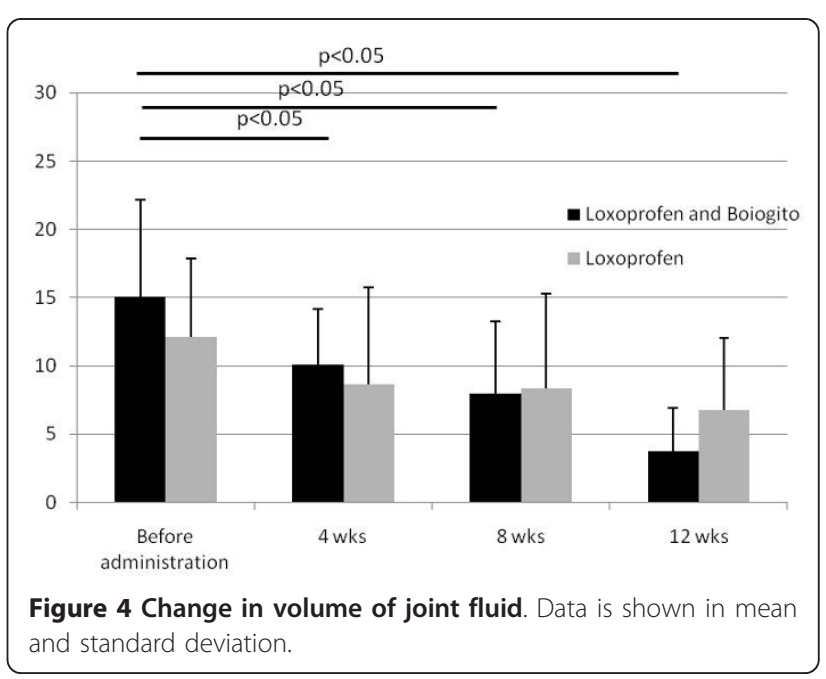


Based on these results, Boiogito was suggested as effective in the conservative treatment for OA of the knee. Boiogito and loxoprofen treatment improve pain and joint effusion, however, their SF-36 scores about another physical area (role physical, bodily pain and general health) were not improve significantly. On the other hand, specific evaluation for knee function according to Knee Society Rating System showed significant difference. These results may be attributed that SF-36 is comprehensive scale and not disease specific scale.

Since herbal medicines consist of numerous components instead of a single component, the mechanisms of decreasing the amount of joint effusion are still unknown. However, with respect to the pharmacological action of Boiogito, effectiveness of sinomenine for inflammation which is a component included in the drug substance was reported in the adjuvant arthritis model [15]. It was assumed that sinomenine inhibits inflammation of secondary synovitis in OA of the knee. It have been reported that other herbal medicine containing astragalus have anti-inflammatory activity through decreasing inflammation related cytokine, Tumor Necrosis Factor (TNF) alpha and Interferon gamma in a rat autoimmune myocarditis model, mice dermatitis model and diabetic mice [16-18]. These reports were not studied on osteoarthritis, however, astragalus which is contained in Boiogito have also possibility for decreasing symptoms of osteoarthritis with joint effusion.

Concerning the limitation of the present study, we did not investigate the single administration of Boiogito and also there is a limitation that the number of cases is small. The studies using a larger number of subjects and the studies including analysis of pharmacological actions based on the analysis of the characteristic changes in the joint effusion should be needed. Further, it is necessary to have a long-term assessment including the degree of satisfaction by patients in the general evaluation not only from the local results in the knees.

In conclusion, this is the first report that the herbal remedy "Boiogito" have a possibility of conservative treatment for joint effusion associated with OA of the knee.

\footnotetext{
Author details

'Department of Joint Replacement and Tissue Engineering, Hokkaido University Graduate School of Medicine, Sapporo, Japan. ${ }^{2}$ Department of Orthopedic Surgery, Hokkaido University Graduate School of Medicine, Sapporo, Japan.
}

\section{Authors' contributions}

TM has made substantial contributions to conception and design, acquisition of data, analysis and interpretation of data. TM, MI, YK, TO and DT carried out the treatment and followed up the patient and contributed to acquisition of data. AM conceived of the study, and participated in its design and coordination. All authors read and approved the final manuscript.

\section{Competing interests}

Each author certifies that he has no commercial associations (e.g. consultations, stock ownership, equity interest, patent/licensing arrangements, etc) that might pose a conflict of interest in connection with the submitted article.

Received: 21 April 2011 Accepted: 10 January 2012

Published: 10 January 2012

\section{References}

1. Zhang W, Moskowitz RW, Nuki G, Abramson S, Altman RD, Arden N, Bierma-Zeinstra S, Brandt KD, Croft P, Doherty M, Dougados M, Hochberg M, Hunter DJ, Kwoh K, Lohmander LS, Tugwell P: OARSI recommendations for the management of hip and knee osteoarthritis, Part I: Critical appraisal of existing treatment guidelines and systematic review of current research evidence. OsteoArthritis and Cartilage 2007, 15:981-1000

2. Pelletier JP, Yaron M, Haraoui B, Cohen P, Nahir MA, Choquette D, Wigler I, Rosner IA, Beaulieu AD: Efficacy and safety of diacerein in osteoarthritis of the knee: a double-blind, placebo-controlled trial. Arthritis Rheum 2000, 43(10):2339-48.

3. Dougados $M$, Nguyen M, Berdah L, Mazieres B, Vignon E, Lequesne M: Evaluation of the structure-modifying effects of diacerein in hip osteoarthritis: ECHODIAH, a three-year, placebo-controlled trial Evaluation of the Chondromodulating Effect of Diacerein in $\mathrm{OA}$ of the Hip. Arthritis Rheum 2001, 44(11):2539-47.

4. Nguyen M, Dougados M, Berdah L, Amor B: Diacerein in the treatment of osteoarthritis of the hip. Arthritis Rheum 1994, 37(4):529-36.

5. Liu L, Resch K, Kaever V: Inhibition of lymphocyte proliferation by the anti-rheumatic drug sinomenine. Int J Immunopharmac 1994, 16:685-691.

6. Liu L, Riese J, Resch K, Kaever V: Impairment of macrophage eicosanoid and nitric oxide production by sinomenine, an alkaloid from Sinomenium acutum. Arznein forsch/Drug Res 1994, 44:1223-1226.

7. Kellgren JH, Lawrence JS: Radiological assessment of osteo-arthrosis. Ann Rheum Dis 1957, 16(4):494-502.

8. Insall JN, Dorr LD, Scott RD, Scott WN: Rationale of the Knee Society clinical rating system. Clin Orthop Relat Res 1989, 248:13-4.

9. Moskowitz RW, Abramson SB, Berenbaum F, Simon LS, Hochberg M: Coxibs and NSAIDs - is the air any clearer? Perspectives from the OARSI/ International COX-2 Study Group Workshop 2007, Osteoarthritis Cartilage 2007, 15:849-856.

10. Chou R, Helfand M, Peterson K, Dana T, Roberts C: Comparative Effectiveness and Safety of Analgesics for Osteoarthritis. Comparative Effectiveness Revie No. 4., (Prepared by the Oregon Evidence-based Practice Center under Contract No. 290-02-0024)Rockville, MD: Agency for Healthcare Research and Quality Available at:www.effectivehealthcare.ahrq gov/reports/final.cfm.

11. Mastbergen SC, Jansen NW, Bijlsma JW, Lafeber FP: Differential direct effects of cyclo-oxygenase-1/2 inhibition on proteoglycan turnover of human osteoarthritic cartilage: an in vitro study. Arthritis Research \& Therapy 2006, 8(1):R2.

12. Smith RL, Kajiyama G, Lane NE: Nonsteroidal antiinflammatory drugs: effects on normal and interleukin 1 treated human articular chondrocyte metabolism in vitro. J Rheumatol 1995, 22:1130-1137.

13. Struglics A, Larsson S, Pratta MA, Kumar S, Lark MW, Lohmander LS: Human osteoarthritis synovial fluid and joint cartilage contain both aggrecanase and matrix metalloproteinase-generated aggrecan fragments. Osteoarthritis Cartilage 2006, 14(2):101-13.

14. Salinardi BJ, Roush JK, Schermerhorn T, Mitchell KE: Matrix metalloproteinase and tissue inhibitor of metalloproteinase in serum and synovial fluid of osteoarthritic dogs. Vet Comp Orthop Traumatol 2006, 19(1):49-55.

15. Liu L, Buchner E, Beitze D, Schmid-Weber CB, Kaever V, Emmrich F, Kinne RW: Amelioration of rat experimental arthritides by treatment with the alkaloid sinomenine. International Journal of Immunopharmacology 1996, 18(10):529-543

16. Lee SJ, Oh SG, Seo SW, Ahn HJ, Geum D, Cho JJ, Park CS: Oral administration of Astragalus membranaceus inhibits the development of 
DNFB-induced dermatitis in NC/Nga mice. Biol Pharm Bull 2007, 30(8):1468-71

17. Zhao P, Su G, Xiao X, Hao E, Zhu X, Ren J: Chinese medicinal herb Radix Astragali suppresses cardiac contractile dysfunction and inflammation in a rat model of autoimmune myocarditis. Toxicol Lett 2008, 182(1-3):29-35.

18. Hoo RLC, Wong JYL, Qiao CF, Xu A, Xu HX, Lam KSL: The effective fraction isolated from Radix Astragali alleviates glucose intolerance, insulin resistance and hypertriglyceridemia in $\mathrm{db} / \mathrm{db}$ diabetic mice through its anti-inflammatory activity. Nutrition \& Metabolism 2010, 7:67-79.

doi:10.1186/1758-2555-4-3

Cite this article as: Majima et al.: Effect of the Japanese herbal

medicine, Boiogito, on the osteoarthritis of the knee with joint effusion. Sports Medicine, Arthroscopy, Rehabilitation, Therapy \& Technology 2012 4:3.

\section{Submit your next manuscript to BioMed Central} and take full advantage of:

- Convenient online submission

- Thorough peer review

- No space constraints or color figure charges

- Immediate publication on acceptance

- Inclusion in PubMed, CAS, Scopus and Google Scholar

- Research which is freely available for redistribution

Submit your manuscript at www.biomedcentral.com/submit 